(C) IEEE. Personal use of this material is permitted. However, permission to reprint/republish this material for advertising or promotional purposes or for creating new collective works for resale or redistribution to servers or lists, or to reuse any copyrighted component of this work in other works must be obtained from the IEEE.

This material is presented to ensure timely dissemination of scholarly and technical work. Copyright and all rights therein are retained by authors or by other copyright holders. All persons copying this information are expected to adhere to the terms and constraints invoked by each author's copyright. In most cases, these works may not be reposted without the explicit permission of the copyright holder. 


\title{
Pit Pattern Classification using Extended Local Binary Patterns
}

\author{
M. Häfner, A. Gangl, M. Liedlgruber, A. Uhl, A. Vécsei, and F. Wrba
}

\begin{abstract}
In this work we present a method for automated classification of endoscopic images according to the pit pattern classification scheme. Images taken during colonoscopy are transformed using a modified version of the Local Binary Patterns operator (LBP). Then, two-dimensional histograms based on the LBP data from different color channels are created. Finally, the classification is carried out by employing the nearest-neighbors (1-NN) classifier in conjunction with the Bhattacharyya distance metric.

The experimental results show that the extended LBP operator delivers superior results and an automated classification of endoscopic images based on the pit pattern classification scheme is feasible.
\end{abstract}

Index Terms-Colonoscopy, colon cancer, local binary patterns, classification

\section{INTRODUCTION}

$\mathbf{T}$ ODAY, the third most common malignant disease in western countries is colon cancer. Therefore a regular colon examination is recommended, especially for people at an age of 50 years and older. Such a diagnosis can be done for example by colonoscopy, which is currently the gold standard for colon cancer detection.

Colonoscopy allows a physician to investigate the inside of the colon by using an endoscope, which is a flexible instrument equipped with a CCD chip for visualization of the organ and controlled by the physician. In case a lesion is detected, tissue samples can be taken and relevant lesions can be removed, avoiding thus surgery.

Modern colonoscopes are able to take pictures from inside the colon. This allows to obtain images for a computer-assisted analysis with the goal of detecting tumorous lesions, which is the aim of this work. To get highly detailed images a special endoscope (magnifying endoscope) is used [1]. Such an endoscope represents a significant advance in colonoscopic diagnosis as it provides images which are up to 150-fold magnified, thus uncovering the fine surface structure of the mucosa as well as small lesions.

In this work we use a modified Local binary patterns operator for an automated classification of visual data acquired by

Manuscript received July 10, 2009; revised September 28, 2009. This work is partially funded by the Austrian Science Fund (FWF) under Project No. L366-N15 and by the Austrian National Bank "Jubiläumsfonds" Project No. 12514 .

Michael Häfner and Alfred Gangl are with the Department of Gastroenterology and Hepatology, Medical University of Vienna, Austria.

Michael Liedlgruber and Andreas Uhl are with the Department of Computer Sciences, Salzburg University, Austria (email: \{mliedl, uhl $\} @$ cosy.sbg.ac.at). Andreas Vécsei is with the St. Anna Children's Hospital, Vienna, Austria. Friedrich Wrba is with the Department of Clinical Pathology, Medical University of Vienna, Austria. a magnifying colonoscope corresponding to different types of lesions. In Section II we review the classification of pit patterns of the colonic mucosa. Section III gives a short introduction to the Local binary patterns transform and existing variants. In Section IV we describe the modified version of LBP and the histogram creation process. The classification process is described in Section V. Experimental results and configuration details of the classification system proposed in this work are presented and discussed in Section VI. Section VII concludes the paper.

\section{Pit Pattern Classification}

Polyps of the colon are a frequent finding and are usually divided into metaplastic, adenomatous, and malignant. As resection of all polyps is time-consuming, it is imperative that those polyps which warrant endoscopic resection can be distinguished: polypectomy of metaplastic lesions is unnecessary and removal of invasive cancer may be hazardous. For these reasons, assessing the malignant potential of lesions at the time of colonoscopy is important.

To be able to differentiate between the different types of lesions a classification method is needed. The most commonly used classification system for distinguishing between nonneoplastic and neoplastic lesions in the colon is the pit pattern classification, originally reported by Kudo et al. [2]. This system allows a differentiation between normal mucosa, hyperplastic lesions (non-neoplastic), adenomas (a pre-malignant condition), and malignant cancer based on the visual pattern of the mucosal surface. Hence, this classification scheme is a convenient tool to decide which lesions need not, which should, and which most likely can not be removed endoscop-

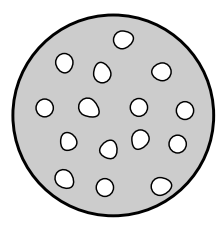

(a) I

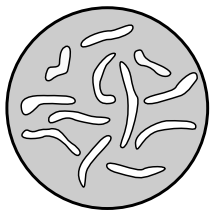

(d) III-L

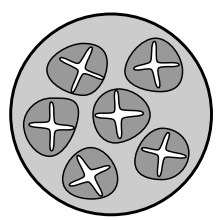

(b) II

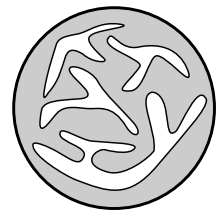

(e) IV

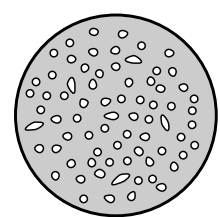

(c) III-S

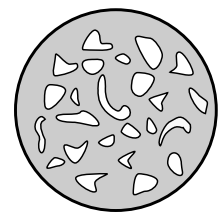

(f) $\mathrm{V}$
Figure 1. Pit pattern classification according to Kudo et al. 


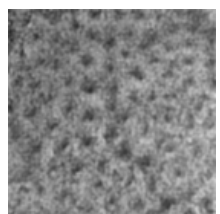

(a) I

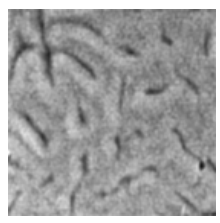

(d) III-L

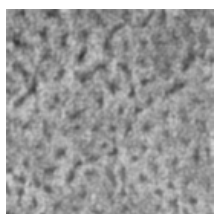

(b) II

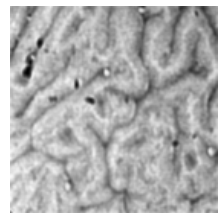

(e) IV

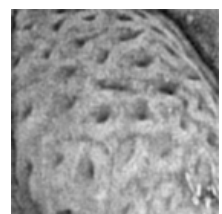

(c) III-S

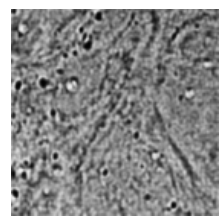

(f) $\mathrm{V}$
Figure 2. Images showing the different types of pit pattern.

ically. The mucosal pattern as seen after dye staining and by using magnification endoscopy shows a high agreement with the histopathologic diagnosis.

As illustrated in Fig. 1, this classification differentiates between five main types according to the mucosal surface of the colon. Type III is divided into types III-S and III-L, designating the size of the pit structure. It has been suggested that type I and II pattern are characteristic of non-neoplastic lesions, type III and IV are found on adenomatous polyps, and type $\mathrm{V}$ are strongly suggestive of invasive carcinoma.

Lesions of type I and II are benign, representing the normal mucosa or hyperplastic tissue, and in fact are non-tumorous. Lesions of type III and IV in contrast represent lesions which are neoplastic. Type $\mathrm{V}$ lesions usually are highly indicative for cancer. Lesions of type I and II can be grouped into nonneoplastic lesions and lesions of type III to V can be grouped into neoplastic lesions. Thus a coarser grouping of lesions into two instead of six classes is possible too.

Using a magnifying colonoscope together with indigo carmine dye spraying, the mucosal crypt pattern on the surface of colonic lesions can be observed [3]. Several studies found a good correlation between the mucosal pit pattern and the histological findings, where especially techniques using magnifying colonoscopes led to excellent results [4].

As depicted in Fig. 1, pit pattern types I to IV can be characterized fairly well, whereas type $\mathrm{V}$ is a composition of unstructured pits. At a first glance this classification scheme seems to be straightforward and easy to be applied. But it needs some experience and exercising to achieve fairly good results [5]. This gets obvious from the example images shown in Fig. 2 (taken out of the training set used throughout this work).

\section{LOCAL BINARY PATTERNS}

Local binary patterns (LBP) [6] are a powerful method to describe local texture properties within an image. In its simplest form, this method compares the grayscale values of the eight nearest neighbors $N_{n}(n=1, \ldots, 8)$ of a pixel $P_{i, j}$ to the grayscale value of the pixel $P_{i, j}$, where $0 \leq i<W$ and $0 \leq j<H$ ( $W$ and $H$ denote the width and the height of the image, respectively). If the value of a neighbor exceeds

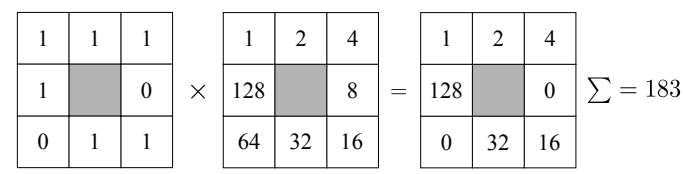

Figure 3. Computation of the LBP number for a given pixel and its neighbors.

the value $P_{i, j}$ the respective neighborhood position is set to 1. The number resulting from the neighborhood bit sequence corresponds to the LBP number. In other words, the neighbors of each pixel are thresholded by the respective center pixel and the resulting binary sequence is used to obtain the final LBP number. This process is repeated for all pixels.

Computing the thresholded neighbor value for the $n$-th neighbor of the pixel located at $(i, j)$ can be written as

$$
T_{n, i, j}=\left\{\begin{array}{ll}
1, & \text { if } N_{n}>P_{i, j} \\
0, & \text { otherwise }
\end{array} .\right.
$$

Based on this, the computation of the LBP number for the pixel $P_{i, j}$ can be formally expressed as

$$
L_{i, j}=\sum_{n=1}^{M} 2^{n-1} T_{n, i, j},
$$

where $M$ denotes the number of neighbors. From (2) it is obvious that the LBP number takes values in the range between 0 and 255. The process of this computation is depicted schematically in Fig. 3.

Since LBP has been introduced the first time, several different variants and extensions have been developed. These variants range from using equidistant neighbors around the center pixel instead of a regular grid [7], over extracting additional information such as contrast (LPB/C) [8] or average neighborhood intensity (LBP/I) [9], to multiresolution approaches, rotation-invariant extensions, and compressed versions. An excellent overview of many variants can be found in [10], [11].

During the last years the LBP operator has already been used throughout several works focused on endoscopic image processing. In [12] the LBP method is compared against other methods for discrimination of gastric polyps in endoscopic videos. This is done by computing 1D histograms based on an LBP transformed image and computing the fraction of LBP values lying between two previously determined threshold values. In [13] the LBP operator is also used for polyp detection. For this purpose the LBP/C operator is used to compute histograms also incorporating contrast information, resulting in two-dimensional histograms. These are fed directly into a support vector machines classifier. The work presented in [14] is targeted at segmentation of color videos resulting from Wireless Capsule Endoscopy (WCE). For this purpose the rotation-invariant LBP operator based on neighbors equidistant around the center pixel has is used to create 3D-histograms. To further reduce the number of possible patterns, only patterns exhibiting a certain amount of uniformity (number of transitions between 0 and 1) are considered for further processing. Then the features are compressed using Principal Component Analysis (PCA). Finally, the LBP features are combined with other features for the segmentation process. 


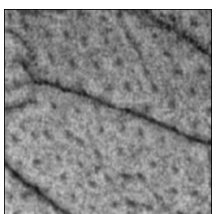

(a)

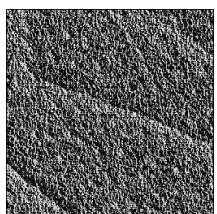

(b)

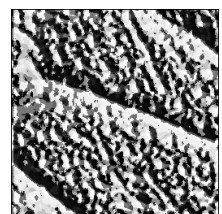

(c)
Figure 4. Differences between different variants of LBP. Original image in (a), standard LBP in (b), and extended LBP in (c).

\section{EXTENDED LOCAL BINARY PATTERNS}

Due to the noisy nature of our image material the standard LBP seems not to be the right choice for our task. This stems from the fact that the LBP operator in its original form is very sensitive to noise. Therefore we use a slightly modified LBP operator, which has already been proposed in a similar way in [15], to overcome this limitation.

Instead of thresholding the neighbors of a pixel with the center pixel $P_{i, j}$, we compute the average over a $B W \times B W$ pixel block centered at $(i, j)$, which we call center block. $B W$ denotes the width of the blocks used. Additionally we compute the averages over all eight $B W \times B W$ blocks, which are adjacent to the center block.

The average value for a pixel block of width $B W$ centered at $(x, y)$ is computed as

$$
B A_{x, y}=\frac{1}{B W^{2}} \sum_{s=-r}^{r} \sum_{t=-r}^{r} I_{x+s, y+t}^{*},
$$

where $r$ denotes the block radius (the maximum horizontal and vertical extent of the block measured from the block center). To be able to compute the LBP value for pixels near or on the border of an image, we use a whole-point symmetrically extended image (horizontal and vertical), which is denoted by $I^{*}$. Using $B A_{x, y}$, the thresholded neighbor value from (1) gets

$$
B T_{n, i, j}=\left\{\begin{array}{ll}
1, & \text { if } B A_{n}>B A_{i, j} \\
0, & \text { otherwise }
\end{array},\right.
$$

where $B A_{n}$ denotes the block average for the $n$-th neighboring block (ordered in a clock-wise fashion). Using $B T_{n, i, j}$, the LBP number based on averaged blocks can be formulated as

$$
L A_{i, j}=\sum_{n=1}^{M} 2^{n-1} B T_{n, i, j} .
$$

This type of LBP operator, which is equivalent to convolving the image with a $B W \times B W$ sized averaging kernel and applying the standard LBP operator by thresholding distant neighbors instead of the adjacent ones, significantly reduces the influence of noise, as shown in Fig. 4.

Once the LBP operator has been applied to all color channels available in the RGB color model, we create twodimensional, normalized histograms which represent the relative co-occurrence frequencies of two different LBP patterns across two distinct color channels (red and blue color component channel in our case). Therefore an entry $H(u, v)$ of such a histogram represents the number of times a pattern $u$ occurs at the same position in the first color channel as pattern $v$ is present in the second color channel, divided by the number of pixels in the image.

\section{Classification}

Once the histograms have been computed, we employ the 1-NN classifier with a suitable histogram distance metric for the classification of unknown images. In our case the distance between two histograms $H_{1}$ and $H_{2}$ is computed by using the discrete Bhattacharyya distance metric, which is defined as

$$
D\left(H_{1}, H_{2}\right)=\sqrt{1-\sum_{i=1}^{N} \sqrt{H_{1, i} H_{2, i}}},
$$

where $N$ denotes the number of bins within the histograms, and $H_{1, i}$ and $H_{2, i}$ denote the values of the $i$-th bin of $H_{1}$ and $H_{2}$, respectively. For comparison purposes we also conducted experiments using the histogram intersection but the Bhattacharyya distance almost always yielded slightly higher classification results. Hence, the results presented in the next section will be based on this metric only.

\section{EXPERIMENTS}

\section{A. Settings}

The image database used throughout our experiments consists of 627 images acquired between the years 2005 and 2008 at the Department of Gastroenterology and Hepatology (Medical University of Vienna) using a zoom-colonoscope (Olympus Evis Exera CF-Q160ZI/L) with a magnification factor set to 150 .

Lesions found during colonoscopy have been examined after application of dye-spraying with indigocarmine as routinely performed in colonoscopy. Biopsies or mucosal resection have been performed in order to get a histopathological diagnosis. Biopsies have been taken from type I, II, and type V lesions, as those lesions need not to be removed or cannot be removed endoscopically. Type III and IV lesions have been removed endoscopically. Out of all acquired images, histopathological classification resulted in 178 non-neoplastic and 449 neoplastic cases. The detailed classification results, which are used as ground truth for our experiments, are shown in Table I.

Using leave-one-out cross-validation, 626 out of 627 images are used as training set. The remaining image is then classified. This process is repeated for each image.

As stated by Ojala et al. in [6], the number of bins used in LBP histograms may be crucial for a system using LBP features. If chosen too low discriminative information may get lost, whereas too many bins may result in sparse and unstable histograms. Throughout this work we used $256^{2}$ bins since this choice produced the best results (tests have been carried out with $32^{2}, 64^{2}, 128^{2}$, and $256^{2}$ bins). Another crucial parameter is $B W$. If chosen too high, discriminative information gets lost. If chosen too low, the influence of noise gets apparent. In our case the optimal value of 5 was found experimentally (out of 3,5,7, and 9).

\begin{tabular}{|l||c|c|c|c|c|c|}
\hline Pit Type & I & II & III-S & III-L & IV & V \\
\hline 2 classes & \multicolumn{2}{|c|}{178} & \multicolumn{5}{|c|}{449} \\
\hline 6 classes & 114 & 64 & 18 & 119 & 232 & 80 \\
\hline \multicolumn{7}{c}{ Table I }
\end{tabular}

NUMBER OF IMAGES PER CLASS USED IN OUR EXPERIMENTS. 


\begin{tabular}{|l|c|c|c|c|c|c||c|}
\hline Pit Type & I & II & III-S & III-L & IV & V & Total \\
\hline \multicolumn{7}{|c|}{ GMRFs with DWT and custom neighborhoods [16] } \\
\hline 2 classes & 90.4 & \multicolumn{5}{|c|}{97.6} & 95.5 \\
\hline 6 classes & 96.4 & 87.5 & 72.2 & 85.7 & 84.9 & 77.5 & 86.1 \\
\hline \multicolumn{7}{|c|}{ Standard LBP } \\
\hline 2 classes & 97.8 & \multicolumn{7}{|c|}{97.6} & 97.6 \\
\hline 6 classes & 99.1 & 93.8 & 77.8 & 94.1 & 94.8 & 93.8 & 94.7 \\
\hline \multicolumn{7}{|c|}{ Extended LBP } \\
\hline 2 classes & 98.3 & \multicolumn{7}{|c|}{99.6} & 99.2 \\
\hline 6 classes & 100.0 & 95.3 & 100.0 & 97.4 & 95.7 & 88.8 & 96.0 \\
\hline
\end{tabular}

Table II

COMPARISON OF CLASSIFICATION RESULTS (GIVEN IN PERCENT) BETWEEN USING GMRFS WITH DWT AND CUSTOM NEIGHBORHOODS [16], STANDARD LBP, AND EXTENDED LBP.

\section{B. Results}

In Table II the results obtained by the proposed method are presented. For comparison purposes we also carried out experiments using the standard LBP operator as presented in [6]. Apart from that we also carried out experiments using a recently published method based on Gaussian Markov random fields (GMRF) applied in the wavelet domain using custom neighborhoods and the 1-NN classifier [16]. The GMRF experiments have been performed using a gaussian blur $(5 \times 5$ kernel with $\sigma=0.5$ ) and CLAHE [17] as preprocessing steps.

The results presented for the LBP tests (standard and extended) have been obtained without any preprocessing. We also carried out experiments with preprocessing (gaussian blur and CLAHE) but this dropped the classification performance. In the case of the extended LBP method this can be explained by the fact that the block averaging already implicitly smooths the images. Thus, an additional gaussian blur results in too much loss of discriminative information.

As we can see from Table II, the proposed method outperforms the GMRF method as well as the standard LBP operator. The overall classification performance of the standard LBP method in the two classes is only slightly higher compared to the GMRF results with an increase of $2.1 \%$, whereas the improvement in the 6-classes cases is considerably high with a gain of $8.6 \%$.

The overall result obtained with the extended LBP method is also higher compared to the GMRF method but only slightly higher compared to the standard LBP method, with a gain of $1.6 \%$ in the 2-classes case and $1.3 \%$ in the 6 -classes case. However, the results for the single classes in the 6-classes case have been improved by between $0.9 \%$ and $22.2 \%$. One exception is class $\mathrm{V}$, for which the result drops by $5 \%$ (most of the wrong classified images in this case are classified as class IV images). The significant boost in case of class III$\mathrm{S}$ can be explained by the rather limited number of images available in this class. Since only 18 images are available one additional correctly classified image improves the result for this class by approximately 5.5\%. For the other classes one correctly classified image contributes between approximately $0.4 \%$ and $1.5 \%$ only to the respective class result.

\section{CONCLUSION}

In this work we have shown, that the extended LBP operator is a simple and very powerful texture feature extraction tool which outperforms the standard LBP operator as well as the method in [16] in terms of classification performance with respect to our endoscopic images. While the extended LBP method outperforms the standard operator only slightly in terms of the overall classification rate, the results obtained for some specific classes in the 6-classes case are considerably higher. Therefore, using the extended LBP operator for a classification of the endoscopic imagery given based on the pit pattern scheme is feasible and delivers superior results.

\section{REFERENCES}

[1] M. J. Bruno, "Magnification endoscopy, high resolution endoscopy, and chromoscopy; towards a better optical diagnosis." Gut, vol. 52, no. 4, pp. 7-11, June 2003.

[2] S.-E. Kudo et al., "Colorectal tumorous and pit pattern," Journal of Clinical Pathology, vol. 47, pp. 880-885, 1994.

[3] S. Kudo et al., "Diagnosis of colorectal tumorous lesions by magnifying endoscopy," Gastrointestinal Endoscopy, vol. 44, no. 1, pp. 8-14, July 1996.

[4] S. Kato, K. Fu, Y. Sano, T. Fujii, Y. Saito, T. Matsuda, I. Koba, S. Yoshida, and T. Fujimori, "Magnifying colonoscopy as a non-biopsy technique for differential diagnosis of non-neoplastic and neoplastic lesions," World Journal of Gastroenterology: WJG, vol. 12, no. 9, pp. 1416-1420, March 2006.

[5] D. Hurlstone, "High-resolution magnification chromoendoscopy: Common problems encountered in "pit pattern" interpretation and correct classification of flat colorectal lesions," American Journal of Gastroenterology, vol. 97, pp. 1069-1070, 2002.

[6] T. Ojala, M. Pietikäinen, and D. Harwood, "A comparative study of texture measures with classification based on featured distributions," Pattern Recognition, vol. 29, no. 1, pp. 51-59, January 1996.

[7] T. Ojala, M. Pietikäinen, and T. Mäenpää, "Multiresolution Gray-Scale and rotation invariant texture classification with local binary patterns," IEEE Transactions on Pattern Analysis and Machine Intelligence, vol. 24, no. 7, pp. 971-987, July 2002.

[8] T. Ojala and M. Pietikäinen, "Unsupervised texture segmentation using feature distributions," Pattern Recognition, vol. 32, no. 3, pp. 477-486, March 1999.

[9] P. Wang, S. Krishnan, C. Kugean, and M. Tjoa, "Classification of endoscopic images based on texture and neural network," in Proceedings of the 23rd Annual International Conference of the IEEE Engineering in Medicine and Biology Society, 2001 (EMBS'01), vol. 4, Istanbul, Turkey, October 2001, pp. 3691-3695.

[10] T. Mäenpää, "The local binary pattern approach to texture analysis extensions and applications," Ph.D. dissertation, University of Oulu, 2003.

[11] T. Mäenpää and M. Pietikäinen, "Texture analysis with local binary patterns," in Handbook of Pattern Recognition and Computer Vision, 3rd ed. World Scientific, 2005, pp. 197-216.

[12] D. K. Iakovidis, D. E. Maroulis, S. A. Karkanis, and A. Brokos, "A comparative study of texture features for the discrimination of gastric polyps in endoscopic video," in Proceedings of the 18th IEEE Symposium on Computer-Based Medical Systems, 2005 (CBMS'05), Dublin, Ireland, June 2005, pp. 575-580.

[13] L. Alexandre, N. Nobre, and J. Casteleiro, "Color and position versus texture features for endoscopic polyp detection," in Proceedings of the International Conference on BioMedical Engineering and Informatics, 2008 (BMEI'08), vol. 2, Sanya, Hainan, China, May 2008, pp. 38-42.

[14] M. Mackiewicz, J. Berens, and M. Fisher, "Wireless capsule endoscopy color video segmentation," IEEE Transactions on Medical Imaging, vol. 27, no. 12, pp. 1769-1781, December 2008.

[15] S. Liao, X. Zhu, Z. Lei, L. Zhang, and S. Li, "Learning multi-scale block local binary patterns for face recognition," in Advances in Biometrics. Springer, 2007, pp. 828-837.

[16] M. Häfner, A. Gangl, M. Liedlgruber, A. Uhl, A. Vecsei, and F. Wrba, "Combining gaussian Markov random fields with the discrete wavelet transform for endoscopic image classification," in Proceedings of the 17th International Conference on Digital Signal Processing (DSP'09), Santorini, Greece, July 2009.

[17] K. Zuiderveld, "Contrast limited adaptive histogram equalization," in Graphics Gems IV, P. S. Heckbert, Ed. Morgan Kaufmann, 1994, pp. 474-485. 